\title{
International Journal of
}

\section{Clinical Obstetrics and Gynaecology}

ISSN (P): 2522-6614

ISSN (E): 2522-6622

(C) Gynaecology Journal

www.gynaecologyjournal.com

2021; 5(4): 225-227

Received: 14-05-2021

Accepted: 17-06-2021

Dr. Saika

Senior Resident, Department of Obstetrics \& Gynecology, GMC,

Rajouri, Jammu and Kashmir, India

Dr. Saba Wani

Senior Resident, Department of Anaesthesia, Rajouri, Jammu and Kashmir, India

\section{Prevalence of hypothyroidism during pregnancy: A prospective study}

\author{
Dr. Saika and Dr. Saba Wani
}

DOI: https://doi.org/10.33545/gynae.2021.v5.i4d.989

Abstract

Aim: To know prevalence of thyroid disorder in pregnancy and pregnancy outcome was done.

Methods: Pregnant cases from Department of Obstetrics and Gynaecology were enrolled in the study during July 2019-January 2020. Detailed history and physical examination was done. Thyroid Function test (FT3, FT4, TSH and Anti-TPO) were performed after the confirmation of pregnancy. Patients were followed up during entire pregnancy. Thirty seven patients dropped out from the study.

Results: In this study, prevalence of thyroid disorder was approximately $27 \%$. Subclinical hypothyroidism was more prevalent and hidden, leading to the poor obstetrical outcome and fetal complications. Rate of miscarriage was high in overt hypothyroid patients.

Conclusion: Due to the immense impact that the maternal thyroid disorder has on maternal and fetal outcome, prompt identification of thyroid disorders and timely initiation of treatment is essential. Thus, universal screening of pregnant women for thyroid disorder should be considered especially in a country like India where there is a high prevalence of undiagnosed thyroid disorder.

Keywords: Hhypothyroidism, overt, subclinical hypothyroidism, thyroid peroxidase (TPO)

\section{Introduction}

The thyroid undergoes physiological changes during pregnancy. Normal pregnancy is associated with an increase in renal iodine excretion, an increase in thyroxine binding proteins, an increase in thyroid hormone production, an increase in HCG level and thyroid stimulatory effects of HCG. All of these factors influence thyroid function tests in pregnant patients. Maternal thyroid function changes during pregnancy and inadequate adaptation to these changes results in thyroid dysfunction. Some of these alterations in thyroid function occur due to increased thyroid hormone-binding globulin (TBG) concentration, increased iodine clearance in the kidneys, and thyrotrophic effect of human chorionic gonadotropin (HCG) ${ }^{[1]}$.

Five factors alter thyroid function in pregnancy: the transient increase in HCG during the first trimester, which stimulate the TSH-R, the estrogen - induced rise in TBG during the first trimester which is sustained during the pregnancy, alteration in immune system, leading to the onset, exacerbation or amelioration of an underlying autoimmune thyroid disease, increased thyroid hormone by the placenta and increased urinary iodide excretion, which can cause impaired thyroid production ${ }^{[2]}$.

The strength of evidence relating maternal hypothyroidism to low IQ in children, strongly suggests that the screening of thyroid function in the early gestation and treatment with Levothyroxine in hypothyroid women would be beneficial. Levothyroxine is the mainstay of treatment for maternal hypothyroidism. The increment of dose adjustment generally is based on the degree of TSH elevation.

Presently there is a lack of information of Indian data on the prevalence and effect of Hypothyroidism in pregnancy. This study was conducted to evaluate the prevalence of hypothyroidism in pregnant women according to new ATA guideline 2017 with maternal and perinatal outcome.

\section{Methodology}

This is a prospective study which was conducted in the Department of Obstetrics and Gynaecology during July 2019-January 2020. 887 Candidates were enrolled after taking informed consents. Pregnant women fulfilling the inclusion criteria were the part of the study. Informed and written consent to participate in this study was obtained.

\section{Dr. Saika}

Senior Resident, Department of Obstetrics \& Gynecology, GMC, Rajouri, Jammu and Kashmir, India 
Detailed history and clinical examination were performed with special regards to maternal age, parity, gestational age, smoking, alcohol consumption or any other addiction, socio-economic status, religion, medical and surgical history. Symptoms of hypothyroidism were asked for past history of hypothyroidism and details of previous thyroxine supplementation was noted and we also looked for the clinical features suggestive of thyroid dysfunction. Serum samples were collected for investigation as specified below including serum TSH , FT3, FT4 estimation which was done by using siemens flex by chemiluminescences immunoassay(CLIA) technique in ADVIA Centaur XP machine using micro plate illuminometers utilizing monoclonal antibody directed against a distinct antigenic determinant on the intact TSH molecule which serves as principle of the Assay .

In women with a raised S.TSH $>4.0 \mathrm{mIU} / \mathrm{L}$ indicating hypothyroidism, as per new 2017 ATA guidelines as mentioned below, the tests for FT3, FT4 and Anti TPO antibody were done. Those with raised TSH but normal FT4 were considered as subclinical hypothyroidism. Women with raised TSH with low FT4 were considered as case of Overt hypothyroidism and the Anti TPO test was done by EUROIMMUN TPO ELISA IgG test kit -human diagnosis, Germany which were first washed in ELISA WASHER and then read on ROBONIK ELISA analyser .The ELISA test kit provides a semi quantities in vitro assay for human autoantibodies of the IgG class against thyroid peroxidase (TPO) in serum or plasma. Values with ratio $>1$ (more than $50 \mathrm{IU} / \mathrm{ml}$ ) were considered as Anti TPO positive and ratio <1(less than $50 \mathrm{IU} / \mathrm{ml}$ ) were considered as Anti TPO Negative. TSH values were repeated every six weeks in patients given levothyroxine for hypothyroidism till TSH values of < $2.5 \mathrm{mIU} / \mathrm{L}$ is attained.

These women were followed during delivery regarding anaemia, preeclampsia, gestational diabetes and obstetric complication such abruption placenta, overall rate of caesarian section, vaginal delivery and post-partum haemorrhage were noted. Neonatal outcomes including incidences of low birth weight, prematurity, intrauterine growth restriction, Apgar score at 1 and 5 minute, neonatal intensive care unit admission and foetal demise were also noted.

Statistical analysis: Descriptive statistics was performed by calculating mean and standard deviation for the continuous variables. Categorical variables are presented as absolute numbers and percentage. The software used for the statistical analysis were SPSS (statistical package for social sciences) version 25.0. Chi-square test was used to investigate whether distributions of categorical variables differ from one another. The $\mathrm{p}$-value was taken significant when less than $0.05(p<0.05)$.

Results: In the present study, 887 pregnant women were screened, out of 887 women 645 (72.72\%) were euthyroid, 147 (16.57\%) women were subclinical hypothyroid and 95(10.71\%) were overt hypothyroids. The prevalence of thyroid disorders in this study was $27.28 \%$ as per table 1 .

Table 1: Prevalence of hypothyroidism in various groups (Distribution of women were done as per the 2017 ATA Guidelines on hypothyroidism in pregnancy).

\begin{tabular}{|c|c|c|c|c|c|}
\hline \multicolumn{5}{|c|}{ No. of subjects } & \\
\hline \multirow{2}{*}{ Euthyroid (72.72\%) } & \multicolumn{2}{|c|}{ Subclinical (16.57\%) } & Overt (10.71\%) & \multirow{2}{*}{ Total } \\
\cline { 2 - 5 } & TPO +ve & TPO -ve & TPO +ve & TPO -ve & \\
\hline $645(72.72 \%)$ & $6(0.68 \%)$ & $141(15.89 \%)$ & $29(3.27 \%)$ & $66(7.44 \%)$ & $887(100 \%)$ \\
\hline
\end{tabular}

On comparison of Anti TPO antibody positivity in the present study we found TPO AB Positivity was significantly more among overt hypothyroidism compared to Sub-clinical hypothyroidism with p value $<0.001$ (statically significant).

On comparison of maternal complications in present study we found Pre-eclampsia/ Eclampsia (9.5\%), PROM (2.7\%) are ssignificantly more among sub-clinical hypothyroid group and similarly Pre-eclampsia/ Eclampsia (22.1\%), Pre-term labour (15.8\%) and PPROM (8.4\%) were significantly more among overt hypothyroidism group compared to control as per figure 1.

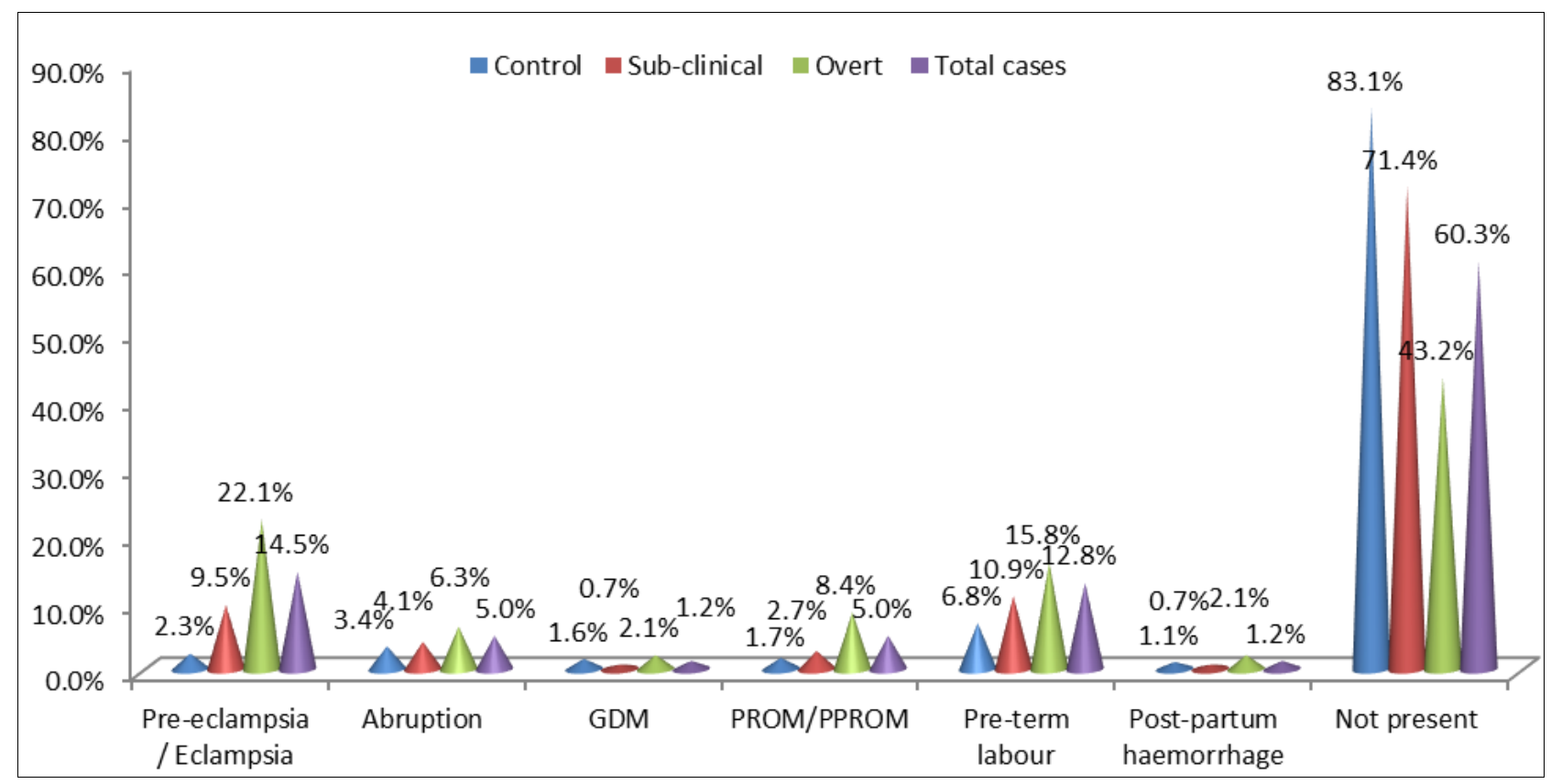

Fig 1: Showing women according to maternal complication in relation to their TSH levels 
Discussion: Among 887 pregnancy women, 72.72\% ( $n=645)$ were euthyroid, $16.57 \% \quad(n=147) \quad$ were subclinical hypothyroidism and $10.71 \%(n=95)$ were overt hypothyroid as per the 2017 ATA guideline on diagnosis of hypothyroid condition among pregnant women. In various studies the prevalence of the overt hypothyroidism is reported in range of $4.8 \%$ to 13.1 in concordance with our present study.

One of the hypothesised reasons for higher mean TSH concentration and range in India compared to western countries may be linked to the country's long-standing iodine deficiency, which has only been partially rectified in the last 20 years by iodization of salt as a national policy. In general hypothyroidisms are concerned with iodine deficiency. Soil degradation with the washing away of iodine from the soil in hilly areas, the use of non-iodinated salts and the ingestion of various goitrogens may also have aggravates the problems. However, even with iodine salt in every household in India, the iodine concentration needed by pregnant women may not be appropriate. The dietary requirement for iodine in pregnant women is $250 \mu \mathrm{g} /$ day, far higher than that fortified by iodinated salt to provide $150 \mu \mathrm{g} /$ day. It may be one of the key reasons for the deficiency of iodine in pregnant women which subsequently leading to hypothyroidism.

The higher prevalence of the hypothyroidism among the pregnant is similar to various studies i.e. Gedam JK et al. 2017 [3], Mandal et.al.2016 ${ }^{[4]}$ and Murthy et al. $2015^{\text {[5] }}$

In controls, 83.1\% women had an uneventful delivery. Among the pregnant women with subclinical hypothyroidism reported pre-term labor in $10.9 \% \quad(n=16)$, followed with preeclampsia/eclampsia in $9.5 \%(n=14)$ and abruption in $4.1 \%$ $(n=6)$. Likewise, in pregnant women with overt hypothyroidism, found to have pre-eclampsia in $22.1 \%(n=21)$ followed by preterm labour in $15.8 \%(n=15)$ and abruption in $6.3 \%(n=6)$ pregnant women. Similarly, the anti-TPO antibody was positive among the mothers who recorded complication with the preeclampsia/eclampsia, followed by pre-term labour and abruption in both the subclinical and overt hypothyroidism cases.

There is a significant maternal complications among the subclinical and overt hypothyroidism compared to the mothers with euthyroid state. Similar to our findings the study conducted by Kiran et al., documented the significant association of pregnancy outcome with the hypothyroidism and TSH levels measured. Some studies demonstrated that TSH $>2.5 \mathrm{uIU} / \mathrm{mL}$ does not have a significant relationship with abortions or any cause of pregnancy loss ${ }^{[6]}$. In study by Gahlawat $\mathrm{P}$ et al., documented a higher rate of abortion among the hypothyroid group of pregnant women compared to euthyroid group similar to our study with abortion among control was $1.9 \%$ and among the pregnant women with hypothyroidism ( $\mathrm{SCH}+$ Overt) was $3.7 \%{ }^{[7]}$.

Thyroid hormones is important in placing and regulating early pregnancy, which could partly explain the association between hypothyroidism and hypertensive gestational disease like preeclampsia, PROM, and preterm birth. The endothelial cell dysfunction, reduction in the local nitric oxide synthesis and the impaired vasodilatation due to reduced circulating thyroid hormone is one of the mechanism of preeclampsia/eclampsia among the hypothyroidism this can further worsen the outcome with abruption, intrauterine growth retardation as secondary to pre-eclampsia and placental insufficiency and pre-term labour and prematurity.

In our study spontaneous abortion is significantly associated with hypothyroid Anti-TPO positive mothers, in concordance to our study several studies have shown that maternal hypothyroidism is associated with increased risk of abortion, stillbirth, premature delivery and hypertension caused by pregnancy ${ }^{[8]}$. Similar to study by various authors there is a significant association of the anti-TPO positive mothers with occurrence of pre-term labour, spontaneous abortion, preeclampsia and eclampsia ${ }^{[9]}$.

\section{Conclusion}

ATA guideline 2011 recommended the trimester specific criteria of TSH estimation in which $2.5 \mathrm{mIU} / \mathrm{L}$ was taken as a cut off for diagnosis of subclinical hypothyroidism. However various review research considered this trimester specific criteria too low leading to over diagnosis and increasing therapeutic burdening of patient with LT4 treatment. Therefore ATA revised their recommendation in 2017 raising S.TSH to $4 \mathrm{mIU} / \mathrm{L}$ as a cut off. Nevertheless, the prevalence of hypothyroidism in pregnancy continued to remain high owing to country's long standing iodine deficiency. Present study strongly recommends routine thyroid screening should be done at the first antenatal visit of every pregnant woman to prevent any obstetric complications in women, growing foetus and event of delivery among the Indian population.

\section{References}

1. Saki F, Dabbaghmanesh MH, Ghaemi SZ, Forouhari S, Omrani GR, Bakhshayeshkaram M. Thyroid Function in Pregnancy and Its Influences on Maternal and Fetal Outcomes. Int J Endocrinol Metab 2014;12(4):e19378.

2. Longo D, Fauci A, Kasper D, Hauser S, Jameson J, Loscalzo J Harrison's Principles of Internal Medicine, 18th ed, McGraw-Hill Professional 2012;1:356-65.

3. Gedam JK, Rajput DA. Prevalence of thyroid disorders among patients attending the antenatal clinic at tertiary care centre, Parel, Mumbai, India.Int J Reprod Contraception, Obstet Gynecol 2017;6(4):1235.

4. Mandal RC, Bhar D, Das A, Basunia SR, Kundu SB, Mahapatra C. Subclinical hypothyroidism in pregnancy: An emerging problem in Southern West Bengal: A crosssectional study. J Nat SciBiol Med 2016;7(1):80-4.

5. Murty N, High prevalence of subclinical hypothyroidism in pregnant women in South India. Int J Reprod Contraception, Obstet Gynecol 2015;4(2):453-7.

6. Khan I, Witczak JK, Hadjieconomou S, Okosieme OE. Preconception thyroid-stimulating hormone and pregnancy outcomes in women with hypothyroidism. Endocr Pract off $\mathrm{J}$ Am Coll Endocrinol Am Assoc Clin Endocrinol 2013;19(4):656-62.

7. Ozdemir H, Akman I, Coskun S, Demirel U, Turan S, Bereket A et al. Maternal Thyroid Dysfunction and Neonatal Thyroid Problems. Int J Endocrinol 2013, 1-6.

8. Tudosa R, Vartej P, Horhoianu I, Ghica C, Mateescu S, Dumitrache I. Maternal and fetal complications of the hypothyroidism-related pregnancy. Maedica (Buchar) 2010;5(2):116-23.

9. Bhattacharyya R, Mukherjee $\mathrm{K}$, Das A, Biswas MR, Basunia SR, Mukherjee A. Anti-thyroid peroxidase antibody positivity during early pregnancy is associated with pregnancy complications and maternal morbidity in later life. J Nat SciBiol Med 2015;6(2):402-5. 